\title{
Particle Dynamics on Snyder space
}

\author{
LEI LU* and A. STERN ${ }^{\dagger}$ \\ Department of Physics and Astronomy, University of Alabama, Tuscaloosa, Al 35487, United States
}

\begin{abstract}
We examine Hamiltonian formalism on Euclidean Snyder space. The latter corresponds to a lattice in the quantum theory. For any given dynamical system, it may not be possible to identify time with a real number parametrizing the evolution in the quantum theory. The alternative requires the introduction of a dynamical time operator. We obtain the dynamical time operator for the relativistic (nonrelativistic) particle, and use it to construct the generators of Poincaré (Galilei) group on Snyder space.
\end{abstract}

*1lv1@crimson.ua.edu

†astern@bama.ua.edu 


\section{Introduction}

Snyder showed long ago that a spatial lattice can be consistent with the continuous symmetries of space-time through the construction of a covariant noncommutative algebra.[1] The algebra is generated by two Lorentz vectors: the time-space coordinate $\hat{x}_{\mu}$, and the energy-momentum $\hat{p}_{\mu}, \mu=0,1,2,3$. The spatial lattice of Snyder, or 'Snyder space', results from the fact that the spatial coordinates $\hat{x}_{i}, i=1,2,3$, have discrete spectra. It differs from a classical lattice because only one coordinate can be determined in a measurement due to the noncommutativity. In a previous article, [2] we found that two distinct representations of the Snyder algebra are possible. They are characterized by an $S U(2)$ quantum number, which takes on integer values for one representation and half-integer values for the other. Continuous symmetry transformations are unitarily implementable on the lattice. The continuous transformations examined in [2] were generated by the momenta and angular momenta, and are associated with the translation and rotation group, respectively. In this article we shall demonstrate, among other things, how to write down the Hermitean generators for the full Poincaré group (Galilei group). This is facilitated by examining the Hamiltonian dynamics of a relativistic particle (nonrelativistic particle) on Snyder space.

Covariance is problematic in the standard Hamiltonian description of a relativistic particle since one treats time differently from the spatial coordinates. It is then not surprising that the Lorentz covariant algebra of Snyder is inconsistent with the Hamiltonian description of a relativistic particle. Various tactics can be taken to get around this issue, and they have been employed in previous articles. These include introducing additional degrees of freedom[3], such as an additional time variable[4], or modifying the particle action[5]. The approach adopted in this article does not require either introducing additional degrees of freedom or deforming the dynamics. Instead, as in the standard Hamiltonian description, we can drop the insistence on covariance, and still have a consistent relativistic particle dynamics. The latter is written down on the Euclidean subalgebra of the Snyder algebra, generated by the spatial components of the four vectors, i.e., $\hat{x}_{i}$ and $\hat{p}_{i} .{ }^{\S}$ Although, $\hat{p}^{0}$ and $\hat{x}^{0}$, no longer appear as independent generators of the algebra in this approach, the notion of energy and time re-appear with the introduction of particle dynamics. One can then derive algebraic properties for these quantities. This is straightforward for the operator $\hat{p}^{0}$ associated with the energy, once we define it by the mass shell condition.

Different approaches can be taken with regard to the time. If one insists, as usual, that time is a real parameter associated with the evolution of the system, then $\hat{p}^{0}$ cannot be the particle Hamiltonian on Snyder space because it will not in general generate time evolution. Rather, the Hamiltonian is some function of $\hat{p}^{0}$. This then implies nonstandard energy-momentum dispersion relations, a feature in common with double special relativity[7]. On the other hand,

\footnotetext{
${ }^{\ddagger}$ As was shown in [2], they are not translations from one point on the lattice to another, but rather are translations in a particular continuous basis of the Hilbert space.

${ }^{\S}$ Some aspects of nonrelativistic dynamics on this space were examined previously in [6].
} 
as we argue here, nonstandard energy-momentum dispersion relations need not be a signature of Snyder space. One can retain the conventional energy-momentum dispersion relation upon re-introducing a time operator. The time operator in this case is not an independent generator of the algebra, but rather a function of the operators $\hat{x}_{i}$ and $\hat{p}_{i}$, along with the evolution parameter denoted by $\lambda$. For this reason we refer to it as a dynamical time operator. The operator is only defined up to constants of the motion, which are not in general central in the algebra. For several examples we determine the definition of the time by requiring it to be a gauge fixing condition in a reparametrization invariant formulation of the dynamics. The choice for the gauge fixing is such that the Euclidean Snyder algebra is realized by the Dirac brackets for the constrained Hamiltonian system. The examples considered in this article are the relativistic and nonrelativistic free particle, along with one-dimensional conservative dynamics. The relativistic (nonrelativistic) free particle action that we start from is Lorentz (Galilei) invariant, as well as reparametrization invariant. We are then able to construct Lorentz (Galilei) boost operators from the Euclidean Snyder algebra which satisfy the Poincaré (Galilei) algebra.

The outline of this article is as follows. In Section 2 we show that the covariant Snyder algebra is inconsistent with the mass shell condition. We then argue that dynamics can be consistently formulated on the Euclidean subalgebra. In Section 3, we write down the symplectic two-form associated with the Euclidean Snyder algebra and develop Hamiltonian dynamics on the resulting phase space. Time is treated as a commuting number in this section, while dynamical time operators are constructed in Section 4. We do this for the three examples of the nonrelativistic and free relativistic particle, and the one-dimensional conservative system. Momentum-dependent eigenfunctions of the time operator, along with space-time symmetry generators, are obtained for the free particle examples. Concluding remarks are made in Section 5.

\section{Covariance lost}

The Lorentz covariant algebra of Snyder is defined by the commutation relations[1]

$$
\begin{aligned}
{\left[\hat{x}_{\mu}, \hat{x}_{\nu}\right] } & =\frac{i}{\Lambda^{2}}\left(\hat{x}_{\mu} \hat{p}_{\nu}-\hat{x}_{\nu} \hat{p}_{\mu}\right) \\
{\left[\hat{x}_{\mu}, \hat{p}_{\nu}\right] } & =i\left(\eta_{\mu \nu}+\frac{\hat{p}_{\mu} \hat{p}_{\nu}}{\Lambda^{2}}\right) \\
{\left[\hat{p}_{\mu}, \hat{p}_{\nu}\right] } & =0
\end{aligned}
$$

where $\mu, \nu, \ldots=0,1,2,3$ and we choose $\left[\eta_{\mu \nu}\right]=\operatorname{diag}(-1,1,1,1)$ for the Minkowski metric. $\Lambda \neq 0$ is the deformation parameter, which has units of energy. The canonical commutation relations of Minkowski space-time coordinates with the four-momenta are recovered in the limit $\Lambda \rightarrow \infty$. The algebra (2.1) was originally obtained starting from the de Sitter group acting on de Sitter 
momentum space, and then projecting to Minkowski space-time. The projection involves a particular coordinatization of de Sitter space by the four-momentum $\hat{p}_{\mu}$, and an identification of four of the de Sitter group generators with the space-time coordinates $\hat{x}_{\mu}$. Following that approach one arrives at an upper bound on the mass, $-\hat{p}^{\mu} \hat{p}_{\mu} \leq \Lambda^{2}$, but this bound is not required if we instead simply postulate the commutation relations (2.1) from the start.

The canonical commutation relations of Minkowski space-time coordinates and the fourmomenta (corresponding to the limit $\Lambda \rightarrow \infty$ ) are inconsistent with the mass shell condition $\hat{p}^{\mu} \hat{p}_{\mu}+m^{2}=0$ defining a relativistic free particle. This is since $\hat{p}^{\mu} \hat{p}_{\mu}$ is not central in the algebra. It is then not surprising that the algebraic relations (2.1) are also inconsistent with the mass shell condition. This is since

$$
\left[\hat{x}_{\mu}, \hat{p}^{\nu} \hat{p}_{\nu}\right]=2 i \hat{p}_{\mu}\left(1+\frac{\hat{p}^{\rho} \hat{p}_{\rho}}{\Lambda^{2}}\right)
$$

Just as one should not insist on Lorentz covariance when writing down the Hamiltonian formalism for a relativistic particle using the canonical commutation relations, one should not insist on Lorentz covariance when writing down relativistic particle dynamics on Snyder space.

Instead, Hamiltonian dynamics for a particle moving in three dimensions is consistently written down on a six-dimensional phase space. By Darboux's theorem we can take the latter to be the Euclidean subalgebra of the Snyder algebra, at least classically. At the quantum level, the algebra is generated by the three spatial components of the coordinates and momenta, $\hat{x}_{i}$ and $\hat{p}_{i}, i=1,2,3$, and defined by the commutation relations

$$
\begin{aligned}
{\left[\hat{x}_{i}, \hat{x}_{j}\right] } & =\frac{i}{\Lambda^{2}} \epsilon_{i j k} \hat{L}_{k} \\
{\left[\hat{x}_{i}, \hat{p}_{j}\right] } & =i\left(\delta_{i j}+\frac{\hat{p}_{i} \hat{p}_{j}}{\Lambda^{2}}\right) \\
{\left[\hat{p}_{i}, \hat{p}_{j}\right] } & =0
\end{aligned}
$$

where $\hat{L}_{i}=\epsilon_{i j k} \hat{x}_{j} \hat{p}_{k}$ are the angular momenta. The three-momenta $\hat{p}_{i}$ are simultaneously diagonalizable and have continuous eigenvalues $p_{i}$. Snyder gave a differential operator representation for $\hat{x}_{i}$ acting on the space of complex functions $\{\phi, \psi, \ldots\}$ of the momentum $\vec{p}=\left(p_{1}, p_{2}, p_{3}\right)$. It is: ${ }^{\pi}$

$$
\hat{x}_{i} \rightarrow i \frac{\partial}{\partial p_{i}}+\frac{i p_{i} p_{j}}{\Lambda^{2}} \frac{\partial}{\partial p_{j}}
$$

These operators are symmetric for the scalar product

$$
(\phi, \psi)=\int d \mu(\vec{p}) \phi(\vec{p})^{*} \psi(\vec{p}),
$$

using the measure

$$
d \mu(\vec{p})=\frac{d^{3} p}{\left(1+\frac{\vec{p}^{2}}{\Lambda^{2}}\right)^{2}}
$$

\footnotetext{
ฯ This was generalized to a one parameter family of representations in [2].
} 
Whereas Snyder's Lorentz covariant algebra (2.1) possesses the independent operators $\hat{x}^{0}$ and $\hat{p}^{0}$, associated respectively with the time and energy, from (2.3) we can define analogous operators as functions of $\hat{x}^{i}$ and $\hat{p}^{i}$ using particle dynamics. For the relativistic free particle, the energy is naturally defined by the mass shell condition

$$
\hat{p}^{0}=\sqrt{\hat{p}_{i} \hat{p}_{i}+m^{2}}
$$

Then

$$
\left[\hat{x}_{i}, \hat{p}^{0}\right]=i \frac{\hat{p}_{i}}{\hat{p}^{0}}\left(1+\frac{\hat{p}_{k} \hat{p}_{k}}{\Lambda^{2}}\right) \quad\left[\hat{p}_{i}, \hat{p}^{0}\right]=0
$$

which differs from the commutation relations for $\hat{p}^{0}$ postulated in (2.1). In the limit $\Lambda \rightarrow \infty$, (2.8) implies the usual Heisenberg equation of motion for a relativistic particle upon identifying $\hat{p}^{0}$ with the Hamiltonian.

As mentioned in the introduction, the notion of time for a relativistic particle can be addressed in different ways. It can be regarded as the evolution parameter associated with the relevant Hamiltonian $H_{t}$, or it can be an operator defined in terms of some evolution generated by the zeroth-component of the four-momentum $\hat{p}^{0}$. Both of these approaches coincide, when one uses the Heisenberg commutation relations, i.e., $\Lambda \rightarrow \infty$, since then $H_{t}=\hat{p}^{0}$. However, this is not true in general, and specifically for commutation relations (2.3). In the former approach, we avoid dealing with a noncommuting time operator. Then $H_{t}$ is some deformation of $\hat{p}^{0}$, which we examine in the following section. The latter approach re-introduces a time operator, which here is a function of the fundamental operators $\hat{x}_{i}$ and $\hat{p}_{i}$, as well as the evolution parameter for the system, which we denote by $\lambda$. As stated in the introduction, the function may be interpreted as a gauge fixing condition starting from a reparametrization invariant action. The commutation relations for this dynamically defined time operator can then be derived using (2.3). The advantage of this approach is that one can retain $\hat{p}^{0}$ as the evolution operator for the relativistic particle [or $\vec{p}^{2} /(2 m)$ as the Hamiltonian for the nonrelativistic particle] and therefore preserve the standard energy-momentum dispersion relations. This appraoch is examined in section four.

\section{Hamiltonian formalism on Snyder space}

\subsection{General formalism}

Before discussing quantum dynamics on the Snyder algebra, we examine classical Hamiltonian dynamics on the associated classical phase space. The phase space is spanned by $x_{i}$ and $p_{i}$, corresponding to the classical analogues of $\hat{x}_{i}$ and $\hat{p}_{i}$. The symplectic two-form for the system is given by

$$
\omega=d x_{i} \wedge d p_{i}-\frac{1}{2} d\left(x_{i} p_{i}\right) \wedge d \ln \left(\vec{p}^{2}+\Lambda^{2}\right)
$$

To see this, we can invert the symplectic matrix to obtain the associated Poisson brackets. The inverse exists because the determinant is $\left(1+\vec{p}^{2} / \Lambda^{2}\right)^{-2}$, which is nonvanishing for finite 
momentum. The resulting Poisson brackets are

$$
\begin{aligned}
\left\{x_{i}, x_{j}\right\} & =\frac{1}{\Lambda^{2}} \epsilon_{i j k} L_{k}, \quad L_{i}=\epsilon_{i j k} x_{j} p_{k} \\
\left\{x_{i}, p_{j}\right\} & =\delta_{i j}+\frac{p_{i} p_{j}}{\Lambda^{2}} \\
\left\{p_{i}, p_{j}\right\} & =0,
\end{aligned}
$$

which are the classical analogues of the commutation relations (2.3). The second term in (3.1) drops out in the limit $\Lambda \rightarrow \infty$, and we recover the canonical symplectic two-form

$$
\omega_{0}=d x_{i} \wedge d p_{i}
$$

More generally, the Darboux map from (3.1) to the canonical symplectic two-form (here written as $\left.d q_{i} \wedge d p_{i}\right)$ is given by

$$
x_{i} \rightarrow q_{i}=x_{i}-\frac{x_{j} p_{j} p_{i}}{\Lambda^{2}+\vec{p}^{2}}
$$

while the momenta $p_{i}$ are invariant under the map. Thus the variables $q_{i}$ are canonically conjugate to $p_{i}$.

For dynamics on the phase space spanned by $x_{i}$ and $p_{i}$, we introduce a Hamiltonian $H$. It generates evolution in some parameter $\lambda$. In general, $\lambda$ need not be the time $t$, but rather some monotonically increasing function of the time. The Hamilton equations of motion

$$
i_{\Delta_{\lambda}} \omega=d H
$$

relate $H$ to the dynamical vector field

$$
\Delta_{\lambda}=\frac{d x_{i}}{d \lambda} \frac{\partial}{\partial x_{i}}+\frac{d p_{i}}{d \lambda} \frac{\partial}{\partial p_{i}}
$$

From the symplectic two-form (3.1), one gets the following equations for the first derivatives of $H$,

$$
\begin{aligned}
\frac{\partial H}{\partial x_{i}} & =-\frac{d p_{i}}{d \lambda}+\frac{\frac{d}{d \lambda}\left(\vec{p}^{2}\right) p_{i}}{2\left(\vec{p}^{2}+\Lambda^{2}\right)} \\
\frac{\partial H}{\partial p_{i}} & =\frac{d x_{i}}{d \lambda}+\frac{\frac{d}{d \lambda}\left(\vec{p}^{2}\right) x_{i}-2 \frac{d}{d \lambda}(\vec{x} \cdot \vec{p}) p_{i}}{2\left(\vec{p}^{2}+\Lambda^{2}\right)}
\end{aligned}
$$

Consistency relations can be obtained by computing second derivatives of $H$.

Aspects of Hamiltonian dynamics on the above phase space were examined previously in [6],[10], along with the equations of motion following from sample Hamiltonians, in particular, the harmonic oscillator Hamiltonian

$$
H_{\mathrm{ho}}=\frac{p^{2}}{2 m}+\frac{1}{2} m \omega^{2} x^{2}
$$


The evolution parameter $\lambda$ was equal to the time in these works. With such an identification, the dynamics generated by $H$ on the phase space associated with symplectic two-form (3.1) is, in general, inequivalent to the dynamics generated by the same Hamiltonian on canonical phase space, the latter defined by the symplectic two-form (3.3). Then, for example, $H_{\text {ho }}$ along with (3.1) does not describe a simple harmonic oscillator, but rather a deformed oscillator. Such a system was examined in [6]. In this article our aim is not to deform, but rather, to preserve the dynamics of any given system while passing from the canonical symplectic two-form (3.3) to $(3.1)$.

To identify the equations of motion (3.5) with some given system, we must specify both $H$ and $\lambda$. Say that the dynamics is Hamiltonian with respect to the canonical symplectic two-form (3.3). That is, it is defined by the symplectic two-form (3.3) and some Hamiltonian $H_{0}$, with the latter generating evolution in the time $t . \|$ Thus the dynamics is given by the standard Hamilton equations

$$
i_{\Delta_{t}} \omega_{0}=d H_{0}
$$

where $\Delta_{t}=\frac{d \lambda}{d t} \Delta_{\lambda}=\frac{d x_{i}}{d t} \frac{\partial}{\partial x_{i}}+\frac{d p_{i}}{d t} \frac{\partial}{\partial p_{i}}$, or in terms of components,

$$
\frac{\partial H_{0}}{\partial x_{i}}=-\frac{d p_{i}}{d t} \quad \frac{\partial H_{0}}{\partial p_{i}}=\frac{d x_{i}}{d t}
$$

Substituting (3.10) into (3.7) gives

$$
\begin{aligned}
& \frac{d \lambda}{d t} \frac{\partial H}{\partial x_{i}}=\frac{\partial H_{0}}{\partial x_{i}}-\frac{p_{i} p_{j}}{\vec{p}^{2}+\Lambda^{2}} \frac{\partial H_{0}}{\partial x_{j}} \\
& \frac{d \lambda}{d t} \frac{\partial H}{\partial p_{i}}=\frac{\partial H_{0}}{\partial p_{i}}-\frac{1}{\vec{p}^{2}+\Lambda^{2}}\left(\left(x_{i} p_{j}-x_{j} p_{i}\right) \frac{\partial H_{0}}{\partial x_{j}}+p_{i} p_{j} \frac{\partial H_{0}}{\partial p_{j}}\right)
\end{aligned}
$$

The conditions state that $H$ generates the same dynamics on the phase space manifold with symplectic two-form (3.1), as $H_{0}$ generates on the phase space manifold with the canonical symplectic two-form (3.3). The equations of motion (3.11) are valid in any number of dimensions. For a given $H_{0}$, one can search for consistent solutions to $H$ and $\frac{d \lambda}{d t}$ as functions of the phase space variables $x_{i}$ and $p_{i}$, and also $\lambda$. These solutions, if they exist, may not be unique. Below we shall obtain solutions in two simple cases: the case of one-dimensional motion and the case of free particle motion.

\subsection{One-dimensional systems}

We first consider the case of motion in one dimension, by which we mean a two-dimensional phase space spanned by $x$ and $p$. Then Eqs. (3.11) simplify to

$$
\frac{d \lambda}{d t} \frac{\partial H}{\partial x}=\frac{\Lambda^{2}}{p^{2}+\Lambda^{2}} \frac{\partial H_{0}}{\partial x}
$$

\footnotetext{
"More generally, it may be possible to have is a solution to (3.7) even when no Hamiltonian description with respect to the canonical symplectic two-form $\omega_{0}$ is possible.
} 


$$
\frac{d \lambda}{d t} \frac{\partial H}{\partial p}=\frac{\Lambda^{2}}{p^{2}+\Lambda^{2}} \frac{\partial H_{0}}{\partial p}
$$

They are satisfied upon writing

$$
H=f\left(H_{0}\right) \quad \frac{d t}{d \lambda}=\left(1+\frac{p^{2}}{\Lambda^{2}}\right) f^{\prime}\left(H_{0}\right),
$$

where $f\left(H_{0}\right)$ is a function of $H_{0}$, and $f^{\prime}\left(H_{0}\right)$ is its derivative. It remains to specify the evolution parameter $\lambda$. Here we should require that $\frac{d t}{d \lambda}$ is everywhere positive for all physical particle trajectories. Of course, this is not a concern if we simply identify $\lambda$ with the time $t$. In that case, from (3.13), we would need $f^{\prime}\left(H_{0}\right)=\left(1+p^{2} / \Lambda^{2}\right)^{-1}$. This can be satisfied when $H_{0}$ depends only on $p$, but it is not in general valid for an arbitrary $x$-dependent Hamiltonian, such as the case with a harmonic oscillator.

Alternatively, instead of trying to identify $t$ with $\lambda$, one can set $H=H_{0}$, and then solve for $\lambda$ as a function of $x, p$ and $t$. This is always possible on the two-dimensional phase space. In that case, one has

$$
H=H_{0} \quad \frac{d t}{d \lambda}=1+\frac{p^{2}}{\Lambda^{2}}
$$

and it follows that $\frac{d t}{d \lambda}$ is everywhere positive.** To obtain the relation between $t$ and $\lambda$ we should integrate (3.14) on the space of solutions: $x=x_{\text {sol }}^{\xi_{1}, \xi_{2}, \ldots}(t), p=p_{\text {sol }}^{\xi_{1}, \xi_{2}, \ldots}(t)$, where $\xi_{1}, \xi_{2}, \ldots$ label the solutions and correspond to constants of motion for the system. Then

$$
\lambda=\Lambda^{2} \int^{t} \frac{d t^{\prime}}{\Lambda^{2}+\left[p_{\mathrm{sol}}^{\xi_{1}, \xi_{2}, \ldots}\left(t^{\prime}\right)\right]^{2}},
$$

from which one can in principle solve for $t$ as a function of $\lambda$ and the phase space variables. Thus $t$ is dynamically determined, and in general will have nontrivial Poisson brackets. However, the latter are not uniquely determined, since one can add a function of the constants of the motion $\xi_{1}, \xi_{2}, \ldots$ to $(3.15)$. The constants of motion are not, in general, central in the algebra, so the commutation relations for the time operator depends on this function. In section 4 , we fix this arbitrariness by demanding that the symplectic structure (3.1) be derived starting from a particle action. We require the action to be reparametrization invariant. The Snyder algebra is then realized by the resulting Dirac brackets for the system upon fixing a gauge condition. The gauge fixing condition then defines the time in terms of the evolution parameter. (This procedure was previously carried out for the case of the relativistic free particle in [8]. Here we also apply it to the nonrelativistic particle and one-dimensional conservative systems. )

\subsection{Free particles}

The problem of finding solutions to (3.11) in more than one dimension is more complicated with the exception of the free particle case, which we now discuss. Here we assume that $H_{0}$ is

\footnotetext{
${ }^{* *}$ We assume that $\Lambda^{2}>0$.
} 
independent of $x_{i}$. (3.11) then implies that $H$ also only depends on the momenta (and possibly $\lambda)$ and the dependence is such that

$$
\frac{d \lambda}{d t} \frac{\partial H}{\partial p_{i}}=\left(\delta_{i j}-\frac{p_{i} p_{j}}{p^{2}+\Lambda^{2}}\right) \frac{\partial H_{0}}{\partial p_{j}}
$$

Upon insisting on rotational invariance, i.e., $H_{0}$ is a function only of $p^{2}$, and the equations simplify further to

$$
\frac{d \lambda}{d t} \frac{\partial H}{\partial p_{i}}=\frac{\Lambda^{2}}{p^{2}+\Lambda^{2}} \frac{\partial H_{0}}{\partial p_{i}}
$$

The solutions are once again (3.13). It again remains to fix $H$ and $\lambda$. Here given $H_{0}$ one can consistently set $t=\lambda$ and solve for $H$ as a function of $p^{2}$. We do this explicitly below for the nonrelativistic and relativistic free particle.

Starting with the nonrelativistic free particle, with $H_{0}=\frac{\vec{p}^{2}}{2 m}$, we get

$$
t=\lambda \quad H=H_{t}=\frac{\Lambda^{2}}{2 m} \ln \left(1+\frac{\vec{p}^{2}}{\Lambda^{2}}\right)
$$

up to constants of integration. Regarding $H_{t}$ as the energy, (3.18) implies a non-standard energy-momentum dispersion relation. Upon expanding $H_{t}$ in $1 / \Lambda$, the standard nonrelativistic kinetic energy appears at zeroth order and the leading correction is fourth order in momentum,

$$
H_{t}=\frac{\vec{p}^{2}}{2 m}-\frac{\left(\vec{p}^{2}\right)^{2}}{4 m \Lambda^{2}}+\cdots
$$

For the case of the relativistic free particle, we can choose $H_{0}=p^{0}$, where

$$
p^{0}=\sqrt{\vec{p}^{2}+m^{2}}
$$

It leads to the equations of motion $d \vec{x} / d t=\vec{p} / p^{0}$. Here $t$ should be interpreted as the zero component of the space-time four vector. Up to constants of integration, the Hamiltonian $H$ which generates the motion on the phase space associated with symplectic two-form (3.1) is

$$
t=\lambda \quad H=H_{t}= \begin{cases}\frac{\Lambda^{2}}{\sqrt{\Lambda^{2}-m^{2}}} \tan ^{-1} \frac{p^{0}}{\sqrt{\Lambda^{2}-m^{2}}}, & m<\Lambda \\ \frac{\Lambda^{2}}{\sqrt{m^{2}-\Lambda^{2}}} \tanh ^{-1} \frac{p^{0}}{\sqrt{m^{2}-\Lambda^{2}}}, & m>\Lambda\end{cases}
$$

(This result appeared previously in [8].) Regarding $H_{t}$ as the energy, we once again get a nonstandard dispersion relation for the energy-momentum for finite $\Lambda$. One recovers $p^{0}$ from (3.21) in the limit $\Lambda \rightarrow \infty$. The leading $1 / \Lambda^{2}$ correction is

$$
\frac{\sqrt{\vec{p}^{2}+m^{2}}}{3 \Lambda^{2}}\left(2 m^{2}-\vec{p}^{2}\right)
$$

which includes a $\frac{2}{3} \frac{m^{3}}{\Lambda^{2}}$ correction to the rest mass energy.

For both of the above free particle solutions, time remains a commutative parameter in the quantum theory. It, along with operator analogue $\hat{H}_{t}$ of the Hamiltonian $H_{t}$, defines the quantum evolution operator

$$
U(t, 0)=e^{-i \hat{H}_{t} t}
$$


From the evolution operator one recovers the usual equations for the expectation value of the operators. For the nonrelativistic particle, one gets

$$
\frac{d}{d t}<\hat{x}_{i}>=-i<\left[\hat{x}_{i}, \hat{H}_{t}\right]>=\frac{<\hat{p}_{i}>}{m} \quad \frac{d}{d t}<\hat{p}_{i}>=0,
$$

while for the relativistic particle

$$
\frac{d}{d t}<\hat{x}_{i}>=-i<\left[\hat{x}_{i}, \hat{H}_{t}\right]>=<\frac{\hat{p}_{i}}{\hat{p}^{0}}>\quad \frac{d}{d t}<\hat{p}_{i}>=0,
$$

where $\hat{p}^{0}$ is defined in (2.7).

Unlike in the above, generic solutions to (3.11) will have $\frac{d \lambda}{d t} \neq 1$, and so then time cannot be identified with the evolution parameter, either in the classical or the quantum theory. In that case, time is a function of the position and momenta, as well as $\lambda$, in the classical theory, while it is an operator in the corresponding quantum theory. We examine this possibility, specifically for the case $H=H_{0}$, in the next section.

\section{Dynamical time operators}

In this section we choose $H$ to be the Hamiltonian $H_{0}$ which generates evolution on the canonical phase space. By so doing, we avoid nonstandard energy-momentum dispersion relations, such as in (3.18) and (3.21). We obtain the time $t$ as a function of $\vec{x}, \vec{p}$ and $\lambda$ in the classical theory for three examples, the free nonrelativistic particle, the free relativistic particle and one-dimensional conservative systems. We do this by demanding that it corresponds to a gauge fixing condition for a reparametrization invariant action.

\subsection{Nonrelativistic free particle}

Since the momentum $\vec{p}$ is constant for free particle trajectories, (3.14) implies that $t$ is a rescaling of $\lambda$ by a constant factor. From (3.15) one gets

$$
\lambda=\frac{\Lambda^{2} t+C}{\vec{p}^{2}+\Lambda^{2}}
$$

along any trajectory. $C$ is some function of the constants of motion, which here include the Galilei boosts

$$
\vec{G}=t \vec{p}-m \vec{x}
$$

as well as $\vec{p}$ and $\vec{L}$. Below we shall show that if we make the choice $C=\vec{p} \cdot \vec{G}$, and hence

$$
t=\lambda+\frac{m \vec{x} \cdot \vec{p}}{\vec{p}^{2}+\Lambda^{2}}
$$

that we recover the full Galilei symmetry for the nonrelativistic free particle and, in particular, $G_{i}$ satisfy the algebra of Galilei boosts. 
Galilei invariance is, of course, insured if one starts with the usual free particle action $S_{\mathrm{nr}}^{0}=\int d t \frac{m}{2}\left(\frac{d \vec{x}}{d t}\right)^{2}$. The action can be rewritten so that it possesses a reparametrization symmetry. Following [11], one can preserve the Galilei invariance, while introducing a gauge degree of freedom $t(\lambda)$ associated with the time [where $\lambda$ is an evolution parameter with no a priori relation to $t$. The corresponding action is

$$
S_{\mathrm{nr}}=\int d \lambda \frac{m}{2} \frac{\dot{\vec{x}}^{2}}{\dot{t}},
$$

the dot denoting differentiation in $\lambda$. If $p_{i}$ denotes the momenta conjugate to $x_{i}$ and $\pi_{t}$ denotes the momentum conjugate to $t$ one gets

$$
p_{i}=m \frac{\dot{x}_{i}}{\dot{t}} \quad \pi_{t}=-\frac{m}{2} \frac{\dot{\vec{x}}^{2}}{\dot{t}^{2}}
$$

and hence the Hamiltonian constraint

$$
\Psi_{1}=\vec{p}^{2}+2 m \pi_{t} \approx 0
$$

generating the reparametrization symmetry $\lambda \rightarrow \lambda^{\prime}(\lambda)$. The symmetry is eliminated upon identifying $\lambda$ with the time $t$, in which case (4.4) reduces to $S_{\mathrm{nr}}^{0}$. Here let us instead use (4.3) to relate $\lambda$ to the time $t$. That is, we impose the gauge constraint

$$
\Psi_{2}=t-\lambda-\frac{m \vec{x} \cdot \vec{p}}{\vec{p}^{2}+\Lambda^{2}} \approx 0
$$

It is straightforward to show that the resulting Dirac brackets for $x_{i}$ and $p_{i}$ are (3.2), yielding the Euclidean Snyder algebra. The Dirac brackets of the time $t$ with $x_{i}$ and $p_{i}$ are

$$
\left\{x_{i}, t\right\}_{D B}=\frac{m x_{j}}{\Lambda^{2}}\left(\delta_{i j}-\frac{2 p_{i} p_{j}}{\Lambda^{2}+\vec{p}^{2}}\right) \quad\left\{p_{i}, t\right\}_{D B}=-\frac{m p_{i}}{\Lambda^{2}}
$$

Since the action (4.4) is Galilei invariant and leads to the Snyder algebra when imposing the gauge fixing (4.7), Galilei invariance must follow when defining the dynamical time for the Snyder algebra according to (4.3).

Next we promote the classical time $t$ to a Hermitean operator $\hat{t}_{\mathrm{nr}}$. Up to operator ordering ambiguities, it is

$$
\hat{t}_{\mathrm{nr}}=\lambda+\frac{1}{2}\left(\hat{x}_{i} \hat{p}_{i} f_{\mathrm{nr}}\left(\overrightarrow{\hat{p}}^{2}\right)+f_{\mathrm{nr}}\left(\overrightarrow{\hat{p}}^{2}\right) \hat{p}_{i} \hat{x}_{i}\right)
$$

where

$$
f_{\mathrm{nr}}\left(\overrightarrow{\hat{p}}^{2}\right)=m\left(\Lambda^{2}+\vec{p}^{2}\right)^{-1}
$$

Since the time operator depends on $\hat{x}_{i}$ and $\hat{p}_{i}$, it is dynamically determined. From (2.3), we derive the following commutation properties for the dynamical time operator:

$$
\left[\hat{x}_{i}, \hat{t}_{\mathrm{nr}}\right]=\frac{i}{\Lambda^{2}}\left(m \hat{x}_{i}-\hat{x}_{j} \hat{p}_{j} \hat{p}_{i} f_{\mathrm{nr}}\left(\overrightarrow{\hat{p}}^{2}\right)-f_{\mathrm{nr}}\left(\overrightarrow{\hat{p}}^{2}\right) \hat{p}_{i} \hat{p}_{j} \hat{x}_{j}\right)
$$




$$
\left[\hat{p}_{i}, \hat{t}_{\mathrm{nr}}\right]=-\frac{i m}{\Lambda^{2}} \hat{p}_{i}
$$

which are the quantum analogues of (4.8). It follows that $\hat{t}_{\mathrm{nr}}$ is rotationally invariant, $\left[\hat{L}_{i}, \hat{t}_{\mathrm{nr}}\right]=$ 0. The commutation relations (4.11) differ from those postulated by Snyder for $\hat{x}^{0}$ in (2.1), which is obvious since Lorentz covariance is not present in (4.11).

The quantum analogue of $(4.2)$ is

$$
\hat{G}_{i}=\frac{1}{2}\left(\hat{t}_{\mathrm{nr}} \hat{p}_{i}+\hat{p}_{i} \hat{t}_{\mathrm{nr}}\right)-m \hat{x}_{i}
$$

$\hat{G}_{i}$ satisfy the algebra of Galilei boosts

$$
\begin{aligned}
{\left[\hat{G}_{i}, \hat{p}_{j}\right] } & =-i m \delta_{i j} \\
{\left[\hat{G}_{i}, \hat{L}_{j}\right] } & =i \epsilon_{i j k} G_{k} \\
{\left[\hat{G}_{i}, \hat{H}_{0}\right] } & =-i \hat{p}_{i}
\end{aligned}
$$

Moreover, the full Galilei algebra is recovered upon including the generators $\hat{p}_{i}, \hat{L}_{i}$ and the Hamiltonian

$$
\hat{H}_{0}=\frac{\hat{p}_{i} \hat{p}_{i}}{2 m}
$$

Here, in addition to (4.13), one has the commutation relations

$$
\begin{aligned}
{\left[\hat{L}_{i}, \hat{p}_{j}\right] } & =i \epsilon_{i j k} p_{k} \\
{\left[\hat{L}_{i}, \hat{L}_{j}\right] } & =i \epsilon_{i j k} \hat{L}_{k},
\end{aligned}
$$

and $\left[\hat{p}_{i}, \hat{p}_{j}\right]=\left[\hat{p}_{i}, \hat{H}_{0}\right]=\left[\hat{L}_{i}, \hat{H}_{0}\right]=\left[\hat{G}_{i}, \hat{G}_{j}\right]=0$. The Galilei group acts unitarily in the quantum theory. The unitary actions of $\hat{p}_{i}$ and $\hat{L}_{i}$ were given explicitly in [2], and using them one can construct the remaining Galilei transformations. This follows because the remaining generators $\hat{G}_{i}$ and $\hat{H}_{0}$ are written in terms of $\hat{x}_{i}$ and $\hat{p}_{i}$ which have a well-defined action on the states.

As $\hat{t}_{\mathrm{nr}}$ is noncommuting, care must be taken in writing down a time-evolution operator in the quantum system. It is constructed using the Hamiltonian (4.14) according to

$$
\tilde{U}(\lambda, 0)=e^{-i \hat{H}_{0} \lambda}
$$

The evolution of the mean values of the space and time coordinates is determined from

$$
\begin{aligned}
\frac{d}{d \lambda}\left\langle\hat{x}_{i}\right\rangle & =-i<\left[\hat{x}_{i}, \hat{H}_{0}\right]>=\left\langle\frac{\hat{p}_{i}}{m}\left(1+\frac{\overrightarrow{\hat{p}}^{2}}{\Lambda^{2}}\right)\right\rangle \\
\frac{d}{d \lambda}\left\langle\hat{t}_{\mathrm{nr}}\right\rangle & \left.=-i<\left[\hat{t}_{\mathrm{nr}}, \hat{H}_{0}\right]\right\rangle+\left\langle\frac{\partial \hat{t}_{\mathrm{nr}}}{\partial \lambda}\right\rangle=\left\langle 1+\frac{\overrightarrow{\hat{p}}^{2}}{\Lambda^{2}}\right\rangle
\end{aligned}
$$


and so the mean velocity is

$$
\frac{d<\hat{x}_{i}>}{d<\hat{t}_{\mathrm{nr}}>}=\frac{\left\langle\frac{\hat{p}_{i}}{m}\left(1+\frac{\overrightarrow{\hat{p}}^{2}}{\Lambda^{2}}\right)\right\rangle}{\left\langle 1+\frac{\overrightarrow{\hat{p}}^{2}}{\Lambda^{2}}\right\rangle},
$$

in contrast to the usual result (3.24). The expression (4.18) reveals the unusual feature that the mean velocity is not linear in the momentum, and that the size and shape of the wavepacket can effect its mean velocity when one approaches the noncommutative scale $\Lambda$. To illustrate this point we can consider a Gaussian distribution in momentum space centered about $\vec{p}=$ $(0,0,<p>)$,

$$
\left.\psi(\vec{p})\right|_{\lambda=0}=\frac{1}{\pi^{3 / 4} \sigma^{3 / 2}}\left(1+\frac{\vec{p}^{2}}{\Lambda^{2}}\right) \exp \left\{-\frac{p_{1}^{2}+p_{2}^{2}+\left(p_{3}-<p>\right)^{2}}{2 \sigma^{2}}\right\}
$$

The factor $1+\frac{\vec{p}^{2}}{\Lambda^{2}}$ was included to cancel out the momentum-dependent factor in the measure (2.6), and we assume $\sigma>0$. The width in momentum space $\Delta p_{i}$ is $\sigma / \sqrt{2}$ in any direction, $i=1,2,3$. Using the differential representation for $\hat{x}_{i}$ in (2.4), we get that the wavepacket is centered about the origin in position space when $\lambda=0,\left\langle\hat{x}_{i}>\left.\right|_{\lambda=0}=0\right.$. For the width of the wavepacket in the $x_{3}$ direction, we find

$$
\left(\left.\Delta x_{3}\right|_{\lambda=0}\right)^{2}=\frac{1}{2 \sigma^{2}}\left\{\left(1+\frac{<p>^{2}}{\Lambda^{2}}\right)^{2}+\frac{\sigma^{2}}{\Lambda^{2}}\left(1+7 \frac{<p>^{2}}{\Lambda^{2}}\right)+\frac{11 \sigma^{4}}{4 \Lambda^{4}}\right\},
$$

which reduces to the usual value of $1 / \sqrt{2} \sigma$ in the $\Lambda \rightarrow \infty$ limit. The effect of noncommutativity is to increase the spread of the wavepacket in position space. This result is consistent with the modified Heisenberg uncertainty relation. From (4.20) one can check that

$$
\Delta x_{3} \Delta p_{3} \geq \frac{1}{2}\left(1+\frac{<p_{3}^{2}>}{\Lambda^{2}}\right)
$$

which follows from the commutation relations (2.3), is satisfied at $\lambda=0 .^{\dagger \dagger}$ At an arbitrary $\lambda$, we get from (4.17) that

$$
<\hat{x}_{3}>=\left\langle\frac{\hat{p}_{3}}{m}\left(1+\frac{\overrightarrow{\hat{p}}^{2}}{\Lambda^{2}}\right)\right\rangle \lambda=\left(1+\frac{<p>^{2}+\frac{5}{2} \sigma^{2}}{\Lambda^{2}}\right) \frac{<p>}{m} \lambda
$$

\footnotetext{
${ }^{\dagger \dagger}$ We have assumed $\hbar=1$ throughout this article. For more discussion of such uncertainty relations, see $[9],[10]$. Here we get an additional time-energy uncertainty relation

$$
\Delta t_{\mathrm{nr}} \Delta \hat{H}_{0} \geq \frac{m}{\Lambda^{2}}<\hat{H}_{0}>
$$
}

which follows from $\left[\hat{t}_{\mathrm{nr}}, \hat{H}_{0}\right]=\frac{i}{\Lambda^{2}} \overrightarrow{\hat{p}}^{2}$. For the wavepacket (4.19), one gets $\left\langle\hat{t}_{\mathrm{nr}}>\left.\right|_{\lambda=0}=0\right.$ and

$$
\left(\left.\Delta \hat{t}_{\mathrm{nr}}\right|_{\lambda=0}\right)^{2}=\frac{m^{2}}{2 \Lambda^{4}}\left(3+\frac{<p>^{2}}{\sigma^{2}}\right),
$$

after applying the differential representation (2.4). This along with

$$
<\hat{H}_{0}>=\frac{1}{2 m}\left(<p>^{2}+\frac{3 \sigma^{2}}{2}\right), \quad \Delta \hat{H}_{0}=\frac{\sigma}{2 m} \sqrt{\frac{3 \sigma^{2}}{2}+2<p>^{2}}
$$

is consistent with the inequality. 


$$
\left.<\hat{t}_{\mathrm{nr}}\right\rangle=\left\langle 1+\frac{\overrightarrow{\hat{p}}^{2}}{\Lambda^{2}}\right\rangle \lambda=\left(1+\frac{<p>^{2}+\frac{3}{2} \sigma^{2}}{\Lambda^{2}}\right) \lambda
$$

The resulting mean speed of the Gaussian wavepacket as a function of its width and of $<p>$ is

$$
\frac{d<\hat{x}_{3}>}{d<\hat{t}_{\mathrm{nr}}>}=\frac{2+\frac{5 \sigma^{2}}{<p>^{2}+\Lambda^{2}}}{2+\frac{3 \sigma^{2}}{<p>^{2}+\Lambda^{2}}} \frac{<p>}{m}
$$

For $\sigma<<\Lambda$ or $|<p>|$, the mean speed differs from $<p>/ m$ by a the factor of $1+$ $\sigma^{2} /\left(<p>^{2}+\Lambda^{2}\right)$.

Unlike the position operators, the time operator (4.9) has continuous eigenvalues. We denote the latter by $\tau$. Then using the differential representation (2.4), we can solve the eigenvalue equation

$$
\hat{t}_{\mathrm{nr}} \phi_{\tau}(\vec{p}, \lambda)=\tau \phi_{\tau}(\vec{p}, \lambda)
$$

for the eigenfunctions in momentum space $\phi_{\tau}(\vec{p}, \lambda)$, evaluated at some $\lambda$. We get

$$
\phi_{\tau}(\vec{p}, \lambda)=\frac{\Lambda^{2}+p^{2}}{8 \pi^{2} \Lambda^{2} m} p^{\frac{i \Lambda^{2}(\lambda-\tau)}{m}-\frac{3}{2}}
$$

Their normalization is such that

$$
\int d \mu(\vec{p}) \phi_{\tau^{\prime}}(\vec{p}, \lambda)^{*} \phi_{\tau}(\vec{p}, \lambda)=\delta\left(\tau-\tau^{\prime}\right)
$$

To see this, substitute (4.25) in the left-hand side and apply the integration measure (2.6) to get

$$
\frac{\Lambda^{2}}{2 \pi m} \int_{0}^{\infty} d p p^{\frac{i \Lambda^{2}\left(\tau^{\prime}-\tau\right)}{m}-1}
$$

Then after making the change in integration variables from $p$ to $w=\frac{\Lambda^{2}}{m} \ln p$, this becomes $\frac{1}{2 \pi} \int_{-\infty}^{\infty} d w e^{i\left(\tau^{\prime}-\tau\right) w}=\delta\left(\tau-\tau^{\prime}\right)$.

\subsection{Relativistic free particle}

We now repeat the previous analysis for the relativistic case. $\lambda$ is again given by (4.1) along any trajectory, where $C$ is some function of the constants of motion. From the equation of motion $d \vec{x} / d t=\vec{p} / p^{0}$, the constants of motion are now $\vec{p}, \vec{L}$ and

$$
\vec{K}=t \vec{p}-\vec{x} p^{0}
$$

We again define $p^{0}$ using the mass shell condition (3.20). The full Poincaré symmetry of the relativistic particle is recovered for the choice $C=\vec{p} \cdot \vec{K}$, and hence

$$
t=\lambda+\frac{p^{0} \vec{x} \cdot \vec{p}}{\Lambda^{2}+\vec{p}^{2}}
$$


with the constants of motion generating the symmetry. Eq. (4.29), along with the equations of motion, leads to

$$
\frac{d x^{i}}{d \lambda}=\frac{p_{i}}{p^{0}}\left(1+\frac{\vec{p}^{2}}{\Lambda^{2}}\right) \quad \frac{d t}{d \lambda}=1+\frac{\vec{p}^{2}}{\Lambda^{2}}
$$

The second equation indicates that $\lambda$ is a momentum-dependent rescaling of the time $t$ on any particle world line, as noted previously. Eq. (4.29) reduces to (4.3) in the nonrelativistic limit.

Analogous to what happens in the nonrelativistic case, Poincaré symmetry follows because one can write down a reparametrization invariant particle action, which yields the Snyder algebra when imposing (4.29) as a gauge fixing.[8] The action can be taken to be the standard one for a relativistic free particle action

$$
S_{\mathrm{rel}}=-m \int d \lambda \sqrt{-\dot{x}_{\mu} \dot{x}^{\mu}}
$$

with the dot again denoting differentiation in evolution parameter $\lambda$ [which we assume has no a priori relation to $\left.x^{0}\right]$. As is well known, the action leads to the mass shell constraint

$$
\Psi_{1}=p^{\mu} p_{\mu}+m^{2} \approx 0
$$

in the Hamiltonian formalism, with $p_{\mu}$ canonically conjugate to $x^{\mu}$. The constraint generates the reparametrization symmetry, which may be eliminated by imposing an additional constraint on the time $t=x^{0}$. It was shown in [8], that the Euclidean Snyder algebra (3.2), is realized by the Dirac brackets if one instead chooses the gauge fixing condition to be

$$
\Psi_{2}=t-\lambda-\frac{p^{0} \vec{x} \cdot \vec{p}}{\Lambda^{2}+\vec{p}^{2}} \approx 0
$$

Since the action (4.31) is Poincaré invariant and leads to the Snyder algebra when imposing this gauge fixing (4.33), Poincaré invariance must follow when defining the dynamical time for the Snyder algebra according to (4.29). Below we shall show explicitly that this symmetry is generated by $p_{\mu}, L_{i}$ and $K_{i}$.

Up to operator ordering ambiguities, the Hermitean operator $\hat{t}_{\text {rel }}$ associated with $t$ in $(4.29)$ is

$$
\hat{t}_{\text {rel }}=\lambda+\frac{1}{2}\left(\hat{x}_{i} \hat{p}_{i} f_{\text {rel }}\left(\overrightarrow{\hat{p}}^{2}\right)+f_{\text {rel }}\left(\overrightarrow{\hat{p}}^{2}\right) \hat{p}_{i} \hat{x}_{i}\right)
$$

where the function $f_{\text {rel }}\left(\left(\overrightarrow{\hat{p}}^{2}\right)\right.$ is given by

$$
f_{\text {rel }}\left(\overrightarrow{\hat{p}}^{2}\right)=\left(\Lambda^{2}+\overrightarrow{\hat{p}}^{2}\right)^{-1} \hat{p}^{0},
$$

and reduces to (4.10) in the nonrelativistic limit. $\hat{t}_{\text {rel }}$ is dynamically determined since it is a function of $\hat{x}_{i}$ and $\hat{p}_{i}$. It is inequivalent to the time operator $\hat{x}^{0}$ appearing in (2.1). Unlike (2.1), the algebra generated by $\hat{t}_{\mathrm{rel}}, \hat{x}_{i}, \hat{p}_{i}$ and $\hat{p}^{0}$ [given in (2.7))] is not Lorentz covariant. From (2.3), we get the commutation relations

$$
\left[\hat{x}_{i}, \hat{t}_{\mathrm{rel}}\right]=\frac{i}{2 \Lambda^{2}}\left(\hat{p}^{0} \hat{x}_{i}+\hat{x}_{i} \hat{p}^{0}+\frac{\Lambda^{2}-\left(\hat{p}^{0}\right)^{2}-m^{2}}{\Lambda^{2}+\overrightarrow{\hat{p}}^{2}} \frac{\hat{p}_{i} \hat{p}_{j}}{\hat{p}^{0}} \hat{x}_{j}+\hat{x}_{j} \frac{\hat{p}_{j} \hat{p}_{i}}{\hat{p}^{0}} \frac{\Lambda^{2}-\left(\hat{p}^{0}\right)^{2}-m^{2}}{\Lambda^{2}+\overrightarrow{\hat{p}}^{2}}\right)
$$




$$
\begin{aligned}
& {\left[\hat{p}_{i}, \hat{t}_{\mathrm{rel}}\right]=-\frac{i}{\Lambda^{2}} \hat{p}^{0} \hat{p}_{i}} \\
& {\left[\hat{p}^{0}, \hat{t}_{\mathrm{rel}}\right]=-\frac{i}{\Lambda^{2}} \hat{p}_{i} \hat{p}_{i}}
\end{aligned}
$$

The second equation agrees with Snyder's commutator $\left[\hat{p}_{i}, \hat{x}^{0}\right]$, while the remaining two equations differ. The third equation follows from the second using (2.7). The time operator is once again rotationally invariant, $\left[\hat{L}_{i}, \hat{t}_{\text {rel }}\right]=0$.

We recover the Poincaré algebra, despite the lack of covariance. The quantum analogue of $(4.28)$ is

$$
\hat{K}_{i}=\frac{1}{2}\left(\hat{t}_{\mathrm{rel}} \hat{p}_{i}+\hat{p}_{i} \hat{t}_{\mathrm{rel}}-\hat{x}_{i} \hat{p}^{0}-\hat{p}^{0} \hat{x}_{i}\right)
$$

From it we obtain the standard commutation relations for the Lorentz boosts,

$$
\begin{aligned}
{\left[\hat{K}_{i}, \hat{p}^{0}\right] } & =-i \hat{p}_{i} \\
{\left[\hat{K}_{i}, \hat{p}_{j}\right] } & =-i \delta_{i j} \hat{p}^{0} \\
{\left[\hat{K}_{i}, \hat{L}_{j}\right] } & =i \epsilon_{i j k} \hat{K}_{k} \\
{\left[\hat{K}_{i}, \hat{K}_{j}\right] } & =-i \epsilon_{i j k} \hat{L}_{k}
\end{aligned}
$$

Upon including $\hat{p}_{i}, \hat{p}^{0}$ and $\hat{L}_{i}$, we obtain $(4.15)$ and $\left[\hat{p}_{i}, \hat{p}_{j}\right]=\left[\hat{p}_{i}, \hat{p}^{0}\right]=\left[\hat{L}_{i}, \hat{p}^{0}\right]=0$, and hence the Poincaré algebra. The Hilbert space for the theory therefore carries unitary representations of Poincaré group.

Poincaré transformations of space-time are implemented with the action of $\hat{p}_{i}, \hat{p}_{0}, \hat{L}_{i}$ and $\hat{K}_{i}$. To see this we need to insure that the gauge condition (4.29) is preserved while also performing a reparametrization $\lambda \rightarrow \lambda^{\prime}(\lambda)$ in the action (4.31). One implements the Poincaré transformations on space-time, as usual, using commutators with the generators, only here the position operators have discrete spectra. Below we simplify to the classical theory and show that infinitesimal space translations, time translations, rotations and Lorentz boosts are obtained by taking Poisson brackets, respectively, with the Poincaré generators $p_{i}, p_{0}, L_{i}$ and $K_{i}$.

1. Infinitesimal spatial translations leave the four-momenta invariant, while the space-time coordinates of the particle $x^{i}(\lambda)$ and $t(\lambda)$ undergo variations

$$
\delta x^{i}(\lambda)=\epsilon^{i}+\frac{d x^{i}}{d \lambda} \delta \lambda \quad \delta t(\lambda)=\frac{d t}{d \lambda} \delta \lambda,
$$

$\epsilon_{i}$ being infinitesimal parameters. It remains to find how $\delta \lambda$ depends on $\epsilon^{i}$. Using (4.30),

$$
\delta x^{i}(\lambda)=\epsilon^{i}+\frac{p_{i}}{p^{0}}\left(1+\frac{\vec{p}^{2}}{\Lambda^{2}}\right) \delta \lambda \quad \delta t(\lambda)=\left(1+\frac{\vec{p}^{2}}{\Lambda^{2}}\right) \delta \lambda
$$


The infinitesimal reparametrization $\delta \lambda$ is constrained by the requirement that the gauge condition (4.29) is preserved by the variations (4.40), i.e.,

$$
t+\delta t(\lambda)=\lambda+\frac{p^{0}(\vec{x}+\delta \vec{x}(\lambda)) \cdot \vec{p}}{\Lambda^{2}+\vec{p}^{2}}
$$

This gives

$$
\delta \lambda=\frac{p^{0} \vec{\epsilon} \cdot \vec{p}}{\Lambda^{2}+\vec{p}^{2}}
$$

It then follows that the infinitesimal variations (4.40) associated with spatial translations are obtained from Poisson brackets with $p_{i}$,

$$
\begin{aligned}
\delta x^{i}(\lambda) & =\left(\delta_{i j}+\frac{p_{i} p_{j}}{\Lambda^{2}}\right) \epsilon_{j}=\left\{x^{i}, \vec{\epsilon} \cdot \vec{p}\right\} \\
\delta t(\lambda) & =\frac{p^{0} p_{i}}{\Lambda^{2}} \epsilon_{i}=\{t, \vec{\epsilon} \cdot \vec{p}\}
\end{aligned}
$$

2. Infinitesimal time translations also leave the four-momenta invariant, while

$$
\delta x^{i}(\lambda)=\frac{p_{i}}{p^{0}}\left(1+\frac{\vec{p}^{2}}{\Lambda^{2}}\right) \delta \lambda \quad \delta t(\lambda)=\epsilon^{0}+\left(1+\frac{\vec{p}^{2}}{\Lambda^{2}}\right) \delta \lambda,
$$

where $\epsilon^{0}$ is infinitesimal and we again used (4.30). In this case the gauge condition (4.29) is preserved for

$$
\delta \lambda=-\epsilon^{0},
$$

and the infinitesimal variations (4.44) associated with time translations are obtained from Poisson brackets with $p_{0}$,

$$
\begin{aligned}
\delta x^{i}(\lambda) & =-\frac{p_{i}}{p^{0}}\left(1+\frac{\vec{p}^{2}}{\Lambda^{2}}\right) \epsilon^{0}=\left\{x^{i}, \epsilon^{0} p_{0}\right\} \\
\delta t(\lambda) & =-\frac{\vec{p}^{2}}{\Lambda^{2}} \epsilon^{0}=\left\{t, \epsilon^{0} p_{0}\right\}
\end{aligned}
$$

3. Infinitesimal rotations leave $p_{0}$ and $t$ invariant, while

$$
\delta x^{i}=\epsilon_{i j k} \eta^{j} x^{k} \quad \delta p_{i}=\epsilon_{i j k} \eta^{j} p_{k},
$$

where $\eta^{k}$ are infinitesimal. The transformation involves no reparametrizations since the gauge condition (4.29) is rotationally invariant. As usual, (4.47) can be expressed in terms of Poisson brackets with $L_{i}$,

$$
\delta x^{i}=\left\{x^{i}, \vec{\eta} \cdot \vec{L}\right\} \quad \delta p_{i}=\left\{p_{i}, \vec{\eta} \cdot \vec{L}\right\}
$$

4. Finally, infinitesimal Lorentz boosts are of the form

$$
\delta x^{i}(\lambda)=\omega^{0 i} t(\lambda)+\frac{p_{i}}{p^{0}}\left(1+\frac{\vec{p}^{2}}{\Lambda^{2}}\right) \delta \lambda \quad \delta t(\lambda)=\omega^{0 i} x^{i}(\lambda)+\left(1+\frac{\vec{p}^{2}}{\Lambda^{2}}\right) \delta \lambda
$$




$$
\delta p_{i}=-\omega^{0 i} p_{0} \quad \delta p_{0}=-\omega^{0 i} p_{i}
$$

where $\omega^{i 0}$ are infinitesimal. In order for the gauge condition (4.29) to be preserved, one now needs

$$
\delta \lambda=\frac{-\omega^{0 i}}{\Lambda^{2}+\vec{p}^{2}}\left\{\left(\Lambda^{2}-m^{2}\right) x^{i}+\left((t-\lambda)\left(m^{2}-\Lambda^{2}\right)-\lambda\left(p^{0}\right)^{2}\right) \frac{p_{i}}{p^{0}}\right\}
$$

The infinitesimal Lorentz boosts (4.49) can then be obtained by taking Poisson brackets with $K_{i}$ defined in (4.28),

$$
\begin{aligned}
\delta x^{i}(\lambda) & =\omega^{0 j}\left\{t \delta_{i j}-\frac{p_{i} x_{j}}{p^{0}}+\frac{p_{i} p_{j}}{\Lambda^{2}}\left(\lambda+\frac{\left(\Lambda^{2}-m^{2}\right)(t-\lambda)}{\left(p^{0}\right)^{2}}\right)+\frac{m^{2}}{\Lambda^{2}} \frac{p_{i} x_{j}}{p^{0}}\right\}=\left\{x_{i}, \omega^{0 j} K_{j}\right\} \\
\delta t(\lambda) & =\frac{\omega^{0 i}}{\Lambda^{2}}\left\{m^{2} x_{i}+p_{i} p^{0}\left(\lambda+\frac{\left(\Lambda^{2}-m^{2}\right)(t-\lambda)}{\left(p^{0}\right)^{2}}\right)\right\}=\left\{t, \omega^{0 i} K_{i}\right\}
\end{aligned}
$$

in addition to $\delta p_{\mu}=\left\{p_{\mu}, \omega^{0 i} K_{i}\right\}$.

The evolution operator (4.16) can be applied to the relativistic system upon choosing $\hat{H}_{0}$ equal to $\hat{p}^{0}$. Then the mean values of $\hat{x}_{i}$ and $\hat{t}$ evolve according to

$$
\begin{aligned}
& \frac{d}{d \lambda}<\hat{x}_{i}>=-i<\left[\hat{x}_{i}, \hat{p}^{0}\right]>=<\frac{\hat{p}_{i}}{\hat{p}^{0}}\left(1+\frac{\overrightarrow{\hat{p}}^{2}}{\Lambda^{2}}\right)> \\
& \frac{d}{d \lambda}<\hat{t}_{\mathrm{rel}}>=-i<\left[\hat{t}_{\mathrm{rel}}, \hat{p}^{0}\right]>+\left\langle\frac{\partial \hat{t}_{\mathrm{rel}}}{\partial \lambda}\right\rangle=\left\langle\left(1+\frac{\overrightarrow{\hat{p}}^{2}}{\Lambda^{2}}\right)>,\right.
\end{aligned}
$$

giving a mean velocity of

$$
\frac{d<\hat{x}_{i}>}{d<\hat{t}_{\mathrm{rel}}>}=\frac{<\frac{\hat{p}_{i}}{\hat{p}^{0}}\left(1+\frac{\overrightarrow{\hat{p}}^{2}}{\Lambda^{2}}\right)>}{<\left(1+\frac{\overrightarrow{\hat{p}}^{2}}{\Lambda^{2}}\right)>}
$$

This is in contrast with the result in (3.25). So, as in the nonrelativistic case, the mean speed depends on the size and shape of the wavepacket as one approaches the noncommutative scale.

Also as in the nonrelativistic case, the dynamical time operator (4.34) has continuous eigenvalues which we again denote by $\tau$. Using the differential representation (2.4), the momentumdependent eigenfunctions of $\hat{t}_{\text {rel }}$ are

$$
\phi_{\tau}(\vec{p}, \lambda)=\mathcal{M} e^{i \kappa \tau} \frac{\Lambda^{2}+p^{2}}{p^{3 / 2}\left(p^{0}\right)^{1 / 2}}\left\{\frac{m}{p \Lambda}\left(m+p^{0}\right)\right\}^{\frac{i \Lambda^{2}(\tau-\lambda)}{m}}
$$

The factor $\mathcal{M} e^{i \kappa \tau}$ is determined from the orthonormality condition (4.26). After substituting in (4.54) and applying the integration measure (2.6), the left-hand side of (4.26) becomes

$$
4 \pi \Lambda^{4}|\mathcal{M}|^{2} e^{i \kappa\left(\tau-\tau^{\prime}\right)} \int_{0}^{\infty} \frac{d p}{p p^{0}}\left\{\frac{m}{p \Lambda}\left(m+p^{0}\right)\right\}^{\frac{i \Lambda^{2}\left(\tau-\tau^{\prime}\right)}{m}},
$$


which after a change of integration variables from $p$ to $w=\kappa+\frac{\Lambda^{2}}{m} \ln \left\{\frac{m}{p \Lambda}\left(m+p^{0}\right)\right\}$, simplifies to

$$
4 \pi \Lambda^{2}|\mathcal{M}|^{2} \int_{\kappa+\frac{\Lambda^{2}}{m} \ln \left\{\frac{m}{\Lambda}\right\}}^{\infty} d w e^{i\left(\tau-\tau^{\prime}\right) w}
$$

The delta function on the right-hand side of (4.26) is recovered for $\kappa \rightarrow-\infty$ and $|\mathcal{M}|=$ $1 /(2 \sqrt{2} \pi \Lambda)$.

\section{3 $1 \mathrm{D}$ conservative systems}

As an example of an interacting system we examine the simplest case of a one-dimensional conservative system. Following the procedure of the previous examples, we require the Hamiltonian generating dynamics on Snyder space to be identical to the Hamiltonian $H_{0}$ on canonical phase space,

$$
H=H_{0}=\frac{p^{2}}{2 m}+V(x),
$$

and so the evolution parameter $\lambda$ cannot be identified with the time $t$. The relation between the two can once again be regarded as a gauge fixing condition. For this we should start with an action which is reparametrization invariant. Such an action can be written as

$$
S_{\text {con }}=\int d \lambda\left\{\frac{m}{2} \frac{\dot{x}^{2}}{\dot{t}}-V(x) \dot{t}\right\}
$$

with the dot again denoting differentiation in the evolution parameter $\lambda$. It reduces to the stan-

dard action for a conservative system when $\lambda$ equals $t$. The Hamiltonian constraint generating the reparametrization symmetry $\lambda \rightarrow \lambda^{\prime}(\lambda)$ is now

$$
\Psi_{1}=H_{0}+\pi_{t} \approx 0
$$

with $p$ and $\pi_{t}$ canonically conjugate to $x$ and $t$, respectively. Since we are only considering one spatial dimension, the Snyder algebra in this case consists of the single relation between $x$ and $p$

$$
\{x, p\}_{\mathrm{DB}}=1+\frac{p^{2}}{\Lambda^{2}},
$$

which we wish to have realized by the Dirac brackets. Then if $\Psi_{2} \approx 0$ denotes the gauge fixing condition its Poisson brackets should satisfy

$$
\frac{p^{2}}{\Lambda^{2}}=\frac{\left\{x, \Psi_{1}\right\}\left\{\Psi_{2}, p\right\}-\left\{x, \Psi_{2}\right\}\left\{\Psi_{1}, p\right\}}{\left\{\Psi_{1}, \Psi_{2}\right\}}=\frac{-\left\{H_{0}, \Psi_{2}\right\}}{\left\{H_{0}+\pi_{t}, \Psi_{2}\right\}},
$$

or simply

$$
\left\{H_{0}, \Psi_{2}\right\}=\frac{p^{2}}{p^{2}+\Lambda^{2}}\left\{\Psi_{2}, \pi_{t}\right\}
$$

Explicit solutions for $\Psi_{2}$ depend on the form for the potential energy $V(x)$. For the example of a linear potential $V(x)=-F x$, one has

$$
\Psi_{2}=t+\frac{1}{F}\left(\Lambda \tan ^{-1} \frac{p}{\Lambda}-p\right)-\lambda,
$$


up to an additive function of $H_{0}$. Setting $\Psi_{2}$ (strongly) equal to zero, the evolution parameter $\lambda$ ranges over a finite domain of length $\pi \Lambda / F$ for any classical trajectory, $p=p_{\text {sol }}=F t+$ constant. For such trajectories, $\lambda$ and $t$ scale as in (3.14). The Dirac brackets of the time $t$ with $x$ and $p$ resulting from the gauge fixing (4.63) are

$$
\{t, x\}_{\mathrm{DB}}=-\frac{p^{2}}{F \Lambda^{2}} \quad\{t, p\}_{\mathrm{DB}}=0
$$

which then can be promoted to commutation relations in the quantum theory.

Another example is the one-dimensional harmonic oscillator with the standard Hamiltonian (3.8). Now (4.62) has the solution

$$
\Psi_{2}=t+\frac{1}{\omega}\left\{\alpha \tan ^{-1} \alpha m \omega x / p-\tan ^{-1} m \omega x / p\right\}-\lambda, \quad \alpha=\frac{1}{\sqrt{1+\frac{2 m H_{\mathrm{ho}}}{\Lambda^{2}}}}
$$

up to an additive function of $H_{0}$. This agrees with the result of the integration in (3.15) along classical trajectories $x=x_{\text {sol }}=A \sin (\omega t+\phi), p=p_{\text {sol }}=m \omega A \cos (\omega t+\phi)$. Also, it reduces to the gauge fixing (4.7) for the free nonrelativistic particle in the limit $\omega \rightarrow 0$. (This differs from the previous example where the free particle limit $F \rightarrow 0$ was singular.) The resulting expressions for the Dirac brackets of the time $t$ with $x$ and $p$ are

$$
\begin{aligned}
\{t, x\}_{\mathrm{DB}} & =\frac{p \alpha^{2}}{\omega \Lambda^{4}}\left(m \omega x p-\left(\Lambda^{2}+p^{2}\right) \alpha \tan ^{-1} \alpha m \omega x / p\right) \\
\{t, p\}_{\mathrm{DB}} & =\frac{m \alpha^{2}}{\Lambda^{4}}\left(\Lambda^{2}+p^{2}\right)\left(p+m \omega x \alpha \tan ^{-1} \alpha m \omega x / p\right)
\end{aligned}
$$

It is a nontrivial problem to find a quantization of this system. Also, the generalization of these examples to higher dimensions is not immediately obvious.

\section{Discussion}

In section 3, we gave conditions [Cf. (3.11)] for writing down any given dynamical system on the symplectic manifold associated with the Snyder algebra. Such a manifold is characterized by the symplectic two-form (3.1). Solutions, if they exist, may not be unique, and require fixing both the Hamiltonian $H$ and the evolution parameter $\lambda$ in terms of the phase space variables and the time. Solutions were found for the case of free particle. There we could either set the time equal to the evolution parameter $\lambda$, so that it remains a real parameter in the quantum theory, or in an alternative approach, equal to a monotonically increasing function of $\lambda$ and the phase space variables. In the latter, the time gets promoted to a Hermitean operator in the quantum theory. The standard energy-momentum dispersion relations were preserved in the latter approach, and the full Poincaré (Galilei) symmetry group for the relativistic (nonrelativistic) particle was recovered. This was because the system could be derived from a Poincaré (Galilei) invariant action. The introduction of a time operator in quantum theory has 
nontrivial consequences, in particular with regards to causality violation. Although causality violation is a well-known feature of relativistic quantum mechanics, the situation here is more dramatic. From the evolution operator (4.16), we can write down a Green function which takes a state with time eigenvalue $\tau^{\prime}$, associated with evolution parameter $\lambda^{\prime}$, to a state with time eigenvalue $\tau$, associated with evolution parameter $\lambda$. It can be expressed as a function of $\tau, \tau^{\prime}$ and $\lambda-\lambda^{\prime}$ using the time eigenfunctions $\phi_{\tau}(\vec{p}, \lambda)$ [eqs. (4.25) or (4.54)],

$$
G\left(\tau, \tau^{\prime}, \lambda-\lambda^{\prime}\right)=\int d \mu(\vec{p}) \phi_{\tau}(\vec{p}, \lambda)^{*} \phi_{\tau^{\prime}}\left(\vec{p}, \lambda^{\prime}\right) e^{-i\left(\lambda-\lambda^{\prime}\right) H_{0}}
$$

The Green function does vanish when $\tau-\tau^{\prime}>0$ and $\lambda-\lambda^{\prime}<0$, and so admits acausal time evolution in the quantum theory. Causality violation in relativistic particle mechanics is standardly cured by writing down the corresponding quantum field theory. However, this is unlikely to be the case here since the resulting noncommutative field theory is nonlocal.

The question of whether or not there exist solutions to (3.11) when interactions are present can be nontrivial. In Section 3.2, we saw that for the case of one-dimensional systems we cannot in general set the evolution parameter equal to the time. So for general interactions, time is not associated with a commuting parameter in the quantum theory. In Section 4.3, we examined the specific case of an interaction with an external potential in one dimension. The action was written in a reparametrization invariant way and the time was obtained from a gauge fixing condition. Interactions with external potentials in higher dimensions should also be possible. Of particular interest are interactions between relativistic particles, which have been examined in a general context in [12]. A nontrivial co-product was introduced for the purpose of studying multiparticle states in a related work.[13] On the other hand, the treatment of particle interactions (in the absence of field theory) is problematic within

our Hamiltonian framework, due to no interaction theorems.[14] Thus a reasonable direction for studying interactions for the systems discussed here is to develop field theory on Snyder space. Preliminary attempts to write down field theory on Snyder space were given in [15],[16], which rely on star product representations of the Snyder algebra. However, the proposed star products have certain inconsistencies, such as non-associativity. Since Snyder space is, in fact, a quantum lattice, it makes sense to instead consider a lattice field theory. Our work with particle dynamics on the quantum lattice, should serve as the ground work for writing fields on such a lattice.

\section{Acknowledgments}

This work was supported in part by the DOE, Grant No. DE-FG02-10ER41714.

\section{REFERENCES}

[1] H. S. Snyder, "Quantized space-time," Phys. Rev. 71, 38 (1947). 
[2] L. Lu and A. Stern, "Snyder space revisited," Nucl. Phys.B 854, 894 (2012).

[3] G. Jaroszkiewicz, "A dynamical model for the origin of Snyder's quantized spacetime algebra," J. Phys. A: Math. Gen. 28 L343 (1995).

[4] J. M. Romero and A. Zamora, "Snyder noncommutative space-time from two-time physics," Phys. Rev. D 70, 105006 (2004).

[5] R. Banerjee, S. Kulkarni and S. Samanta, "Deformed symmetry in Snyder space and relativistic particle dynamics," JHEP 0605, 077 (2006).

[6] S. Mignemi, "Classical and quantum mechanics of the nonrelativistic Snyder model," Phys. Rev. D84, 025021 (2011); "Classical and quantum mechanics of the nonrelativistic Snyder model in curved space," [arXiv:1110.0201 [hep-th]].

[7] For a recent review see, G. Amelino-Camelia, "Doubly-Special Relativity: Facts, Myths and Some Key Open Issues," Symmetry 2, 230 (2010).

[8] A. Pinzul and A. Stern, "Space-time noncommutativity from particle mechanics," Phys. Lett. B 593, 279 (2004); A. Stern, "Relativistic Particles on Quantum Space-time," Phys. Lett. A 375, 2498 (2011).

[9] A. Kempf, G. Mangano and R. B. Mann, Phys. Rev. D 52, 1108 (1995); A. Kempf and G. Mangano, "Minimal length uncertainty relation and ultraviolet regularization," Phys. Rev. D 55, 7909 (1997).

[10] M. V. Battisti and S. Meljanac, "Modification of Heisenberg uncertainty relations in noncommutative Snyder space-time geometry," Phys. Rev. D 79, 067505 (2009).

[11] R. Banerjee, B. Chakraborty and S. Gangopadhyay, "Noncommutativity and reparametrisation symmetry," J. Phys. A A 38, 957 (2005).

[12] G. Amelino-Camelia, L. Freidel, J. Kowalski-Glikman and L. Smolin, "The principle of relative locality," Phys. Rev. D 84, 084010 (2011).

[13] A. Agostini, G. Amelino-Camelia and F. D'Andrea, "Hopf-algebra description of noncommutative-spacetime symmetries," Int. J. Mod. Phys. A 19, 5187 (2004).

[14] D. G. Currie, T. F. Jordan and E. C. G. Sudarshan, "Relativistic invariance and Hamiltonian theories of interacting particles," Rev. Mod. Phys. 35, 350 (1963); A. P. Balachandran, G. Marmo and A. Stern, "A Lagrangian Approach To The No Interaction Theorem," Nuovo Cim. A 69, 175 (1982).

[15] M. V. Battisti and S. Meljanac, "Scalar Field Theory on Non-commutative Snyder SpaceTime," Phys. Rev. D 82, 024028 (2010).

[16] F. Girelli and E. R. Livine, "Scalar field theory in Snyder space-time: Alternatives," JHEP 1103, 132 (2011). 